\title{
Local production of angiotensin II in the subfornical organ causes elevated drinking
}

\author{
Koji Sakai, ${ }^{1}$ Khristofor Agassandian, ${ }^{2,3}$ Satoshi Morimoto,, ${ }^{1}$ Puspha Sinnayah, ${ }^{2}$ \\ Martin D. Cassell,2,3 Robin L. Davisson, ${ }^{4,5}$ and Curt D. Sigmund1,3,6
}

\begin{abstract}
1Department of Internal Medicine, ${ }^{2}$ Department of Anatomy and Cell Biology, and ${ }^{3}$ Center on Functional Genomics of Hypertension, Roy J. and Lucille A. Carver College of Medicine, University of lowa, lowa City, lowa, USA. ${ }^{4}$ Department of Biomedical Sciences, College of Veterinary Medicine, Cornell University, Ithaca, New York, USA. ${ }^{5}$ Department of Cell and Developmental Biology, Weill Cornell Medical College, Cornell University, New York, New York, USA. ${ }^{\circ}$ Department of Molecular Physiology and Biophysics,
\end{abstract} Roy J. and Lucille A. Carver College of Medicine, University of lowa, lowa City, lowa, USA.

\begin{abstract}
The mechanism controlling cell-specific Ang II production in the brain remains unclear despite evidence supporting neuron-specific renin and glial- and neuronal-specific angiotensinogen (AGT) expression. We generated double-transgenic mice expressing human renin ( $h R E N$ ) from a neuron-specific promoter and human $A G T$ ( $b A G T$ ) from its own promoter (SRA mice) to emulate this expression. SRA mice exhibited an increase in water and salt intake and urinary volume, which were significantly reduced after chronic intracerebroventricular delivery of losartan. Ang II-like immunoreactivity was markedly increased in the subfornical organ (SFO). To further evaluate the physiological importance of de novo Ang II production specifically in the SFO, we utilized a transgenic mouse model expressing a floxed version of $h A G T\left(h A G T^{f l o x}\right)$, so that deletions could be induced with Cre recombinase. We targeted SFO-specific ablation of $h A G T^{f l o x}$ by microinjection of an adenovirus encoding Cre recombinase ( $\mathrm{AdCre}$ ). SRA ${ }^{\text {flox }}$ mice exhibited a marked increase in drinking at baseline and a significant decrease in water intake after administration of $A d C r e /$ adenovirus encoding enhanced GFP (AdCre/AdEGFP), but not after administration of AdEGFP alone. This decrease only occurred when Cre recombinase correctly targeted the SFO and correlated with a loss of hAGT and angiotensin peptide immunostaining in the SFO. These data provide strong genetic evidence implicating de novo synthesis of Ang II in the SFO as an integral player in fluid homeostasis.
\end{abstract}

\section{Introduction}

The renin-angiotensin system (RAS) is known to play a critical role in body fluid regulation through its actions within the kidney and central nervous system. Direct intracranial or intracerebroventricular injection of Ang II causes increases in blood pressure and dipsogenic behavior (1-3). Furthermore, centrally administered Ang II receptor antagonists are known to eliminate the cardiovascular effects caused by exogenous Ang II (4-6). Although these experiments have provided important evidence that the central nervous system is a target for the pressor and dipsogenic actions of exogenous Ang II, it remains unclear how endogenous Ang II is formed within the brain, where it is located, and how regional Ang II production correlates with specific cardiovascular responses.

Renin catalyzes the first of 2 steps in the processing of angiotensinogen (AGT) to Ang II and has been reported to be expressed in numerous tissues, including the brain. Indeed, there is now clear evidence that all components of the RAS are expressed within the brain. AGT exhibits a widespread distribution in astroglia throughout the brain but a much more selective regional distribution in neurons (7-9). Renin was previously identified in neu-

Nonstandard abbreviations used: aCSF, artificial cerebrospinal fluid; AdCre, adenovirus encoding Cre recombinase; AdEGFP, adenovirus encoding EGFP; AGT, angiotensinogen; $\mathrm{AT}_{1}$, Ang II receptor, type 1 ; BW, body weight; CSF, cerebrospinal fluid; EGFP, enhanced GFP; hAGT, human AGT; hREN, human renin; MnPO, median preoptic nucleus; $\mathrm{PVN}$, paraventricular nucleus; RAS, renin-angiotensin system; SFO, subfornical organ; SRA mice, double-transgenic mice expressing $h R E N$ from a neuron-specific promoter and $h A G T$ from its own promoter.

Conflict of interest: The authors have declared that no conflict of interest exists. Citation for this article: J. Clin. Invest. 117:1088-1095 (2007). doi:10.1172/JCI31242. rons $(10,11)$, and using a renin promoter-directed enhanced GFP (EGFP) gene, we reported that renin-expressing cells are located in or near regions controlling cardiovascular function and are primarily neurons $(12,13)$. Ang II, like other neurotransmitters, has been localized in neuronal processes and in the vicinity of nerve terminals (14). That renin and AGT are located in close proximity to Ang II receptor-containing neurons suggests a model for local production and action of Ang II. Renin released from neurons would initiate the processing of AGT released from glial cells (and perhaps neurons), eventually forming Ang II, which binds locally to Ang II receptor-containing neurons. Other evidence supporting the existence of an alternative form of renin in the brain that is not secreted, coupled with the observation that some neurons appear to coexpress both renin and AGT (and angiotensin converting enzyme), suggests the potential for intracellular generation of Ang II (15-18). Whether this Ang II is packaged into secretory or synaptic vesicles has yet to be determined experimentally.

In the brain, the subfornical organ (SFO) has long been known to be an important cardiovascular control region regulating fluid homeostasis in response to Ang II (19). However, the SFO is one of several circumventricular organs that lie outside the blood-brain barrier and thus have access to circulating and intraventricular Ang II. Indeed, systemic Ang II causes drinking that is mediated by the SFO (19-21). This, coupled with the observation that AGT is abundantly expressed in the SFO, suggests the possibility that SFO Ang II may either be derived (taken up) from the circulation or cerebrospinal fluid (CSF) or synthesized locally from AGT (22). Angiotensinergic connections between the SFO and hypothalamic nuclei that control drinking have been described in detail (23-25). 

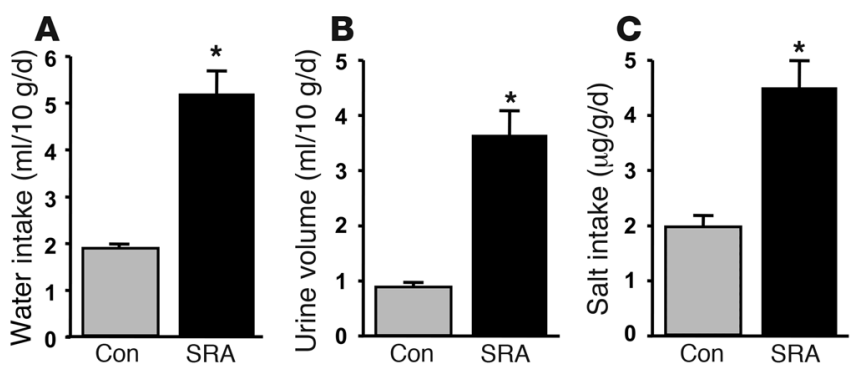

Figure 1

Volume homeostasis in SRA mice. Water intake (A), urine volume (B), and salt intake (C) in SRA mice $(n=14)$ and control littermates (Con; $n=25) .{ }^{*} P<0.001$ compared with control mice.

Consequently, the purpose of this study was to examine the physiological consequences on fluid homeostasis of regional production of Ang II derived from local renin and AGT. Genetic techniques that allow for spatiotemporal control of gene expression and ablation in mice provide innovative tools with which to experimentally dissect complex regulatory systems such as the RAS in the brain $(26,27)$. To test the hypothesis that the SFO plays a role in the dipsogenic actions of Ang II derived from the local synthesis of renin and AGT, we used a combination of cell-specific promoters to drive renin and AGT synthesis (28), an AGT gene modified for cell-specific deletion by Cre recombinase (29-31), and the stereotaxic delivery of adenoviruses encoding Cre recombinase (AdCre) to the SFO $(27,31)$. We report that targeted expression of $h R E N$ and $h A G T$ resulted in a marked increase in water and salt intake, which significantly decreased in response to the blockade of AT1 receptors in the brain. There mice exhibited elevated Ang II specifically in the SFO. SFO-specific ablation of hAGT synthesis by Cre recombinase resulted in a significant decrease in hAGT and Ang II immunostaining in the SFO and correlated with a significant decrease in water intake. These results provide genetic evidence that local de novo synthesis of Ang II in the SFO plays an integral role in regulating fluid hemostasis.

\section{Results}

Our recent studies using a dual-reporter transgenic model in which renin- and $A G T$-expressing cells were identified with EGFP and $\beta$-galactosidase, respectively, indicated close localization of renin- and AGT-containing cells in a number of cardiovascular control regions, with renin primarily localized to neurons $(12,13)$. Therefore, in order to overexpress Ang II in the brain while retaining the normal cellular distribution of Ang II formation, we bred mice expressing human $A G T$ ( $h A G T)$ under the control of its own endogenous promoter with mice expressing human renin ( $h R E N)$ under the control of the neuron-specific synapsin I promoter (SR mice). We previously reported that the source of circulating hAGT in the hAGT mouse is the liver, whereas in the brain, $h A G T$ is expressed in both astrocytes and neurons $(22,32)$. In SR mice, we confirmed that the expression of $h R E N$ in this model is restricted to neurons and is not released into the systemic circulation (28). Because of the species specificity of the renin-AGT enzymatic reaction and the absence of circulating hREN, double-transgenic mice expressing $h R E N$ from a neuron-specific promoter and $h A G T$ from its own promoter (SRA mice) would be expected to have increased synthesis of Ang II specifically in the brain.
We first measured arterial pressure in SRA mice by radiotelemetry and observed a significant increase in arterial pressure $(121 \pm 6$ $\mathrm{mmHg}$ in SRA mice versus $97 \pm 4 \mathrm{mmHg}$ in nontransgenic mice, $n=7 ; P=0.004)$. We then focused entirely on water and electrolyte homeostasis and compared water intake, urinary volume, and salt intake between SRA mice and control littermates. SRA mice exhibited a nearly 3-fold increase in baseline water intake (Figure 1A). Accordingly, there was a marked elevation in the production of urine (Figure 1B), which was osmotically dilute (Table 1). When given a choice of tap water or hypertonic saline, the SRA mice exhibited an increased preference (2.3-fold) for sodium consumption (Figure 1C). To determine whether the increase in drinking was due to an Ang II receptor, type 1-dependent ( $\mathrm{AT}_{1}$-dependent) mechanism, we chronically infused losartan using an icv microinfusion pump in separate groups of SRA and control mice. Chronic icv infusion of losartan significantly reduced water intake, urinary volume, and salt intake in both SRA and control mice (Table 2), but the decrease was significantly greater in double-transgenic SRA mice (Figure 2). Chronic icv infusion of artificial cerebrospinal fluid (aCSF) did not affect water intake $(1.6 \mathrm{ml} / 10 \mathrm{~g}$ body weight $[\mathrm{BW}] / \mathrm{d}$ versus $1.5 \mathrm{ml} / 10 \mathrm{~g} \mathrm{BW} / \mathrm{d} ; P=0.57)$ in control mice. Importantly, chronic subcutaneous infusion of the same dose of losartan did not affect water intake in SRA mice $(5.4 \mathrm{ml} / 10 \mathrm{~g} \mathrm{BW} / \mathrm{d}$ versus $5.1 \mathrm{ml} / 10 \mathrm{~g} \mathrm{BW} / \mathrm{d} ; P=0.95)$.

One of the first phenotypes we observed was a smaller number of weaned SRA offspring than would be expected based on Mendelian inheritance. From a total 2,097 offspring from 123 breeding females, only 259 (in contrast to the expected 524) offspring were genotyped at weaning as double transgenic $\left(P<0.0001, \chi^{2}\right.$ test). There was no apparent difference in the number of nontransgenic $\left(S R^{-} / A^{-}, n=653\right)$ or single-transgenic mice $\left(S R^{-} / A^{+}\right.$or $S R^{+} / A^{-}$, $n=1,185)$ compared with what was expected, and once SRA mice survived to weaning, there was little mortality observed thereafter. Moreover, there was no difference in the litter size of offspring derived from a breeding of SRA mice (5.9 \pm 1.9 pups/litter from 86 litters) compared with other double-transgenic strains, in which no mortality was observed $(6.0 \pm 1.3$ pups per litter from 66 litters; $P=0.72)(33)$. To determine a possible reason for mortality in young SRA mice, we measured sodium and potassium output in the survivors. We observed a significant increase in both sodium and potassium output, which suggested the possibility that newborn SRA mice may have had difficulty concentrating their urine or retaining electrolytes (Table 1 ).

We next performed immunohistochemistry to assay for the location of angiotensin peptide production in the brain of SRA mice. At the regional level, we observed a dramatic increase in Ang II-like immunoreactivity in the SFO of SRA mice (in Figure 3, compare B with $A$ and $E$ with $D$ ). The specificity of the immunohistochemistry was confirmed by preabsorption with the immunizing peptide

\section{Table 1}

Electrolyte homeostasis

\begin{tabular}{lccc}
$\begin{array}{c}\text { Urine osmolality } \\
(\mathbf{m} \mathbf{0 s m} / \mathbf{k g})\end{array}$ & $\begin{array}{c}\mathbf{N a}^{+} \text {excretion } \\
(\mu \mathbf{E q} / \mathbf{1 0} \mathbf{~ g ~ B W / d})\end{array}$ & $\begin{array}{c}\mathbf{K}^{+} \text {excretion } \\
(\mu \mathbf{E q} / \mathbf{1 0} \mathbf{~ g ~ B W / d})\end{array}$ \\
Control $(n=13)$ & $1,769 \pm 138$ & $97 \pm 10$ & $146 \pm 12$ \\
SRA $(n=11)$ & $715 \pm 48^{\mathrm{A}}$ & $182 \pm 11^{\mathrm{A}}$ & $253 \pm 15^{\mathrm{A}}$ \\
\hline
\end{tabular}

AP $<0.01$ versus control mice. 


\section{Table 2}

Fluid homeostasis in SRA mice before and after icv administration of losartan

\begin{tabular}{lrrrr} 
& \multicolumn{2}{c}{ Control $(\boldsymbol{n}=\mathbf{9})$} & \multicolumn{2}{c}{ SRA $(\boldsymbol{n}=6)$} \\
& Pre-Iosartan & Post-Iosartan & Pre-Iosartan & Post-Iosartan \\
$\begin{array}{l}\text { Water intake } \\
(\mathrm{ml} / 10 \mathrm{~g} \mathrm{BW} / \mathrm{d})\end{array}$ & $1.5 \pm 0.13$ & $0.99 \pm 0.19^{\mathrm{A}}$ & $4.5 \pm 0.72$ & $2.0 \pm 0.23^{\mathrm{B}}$ \\
$\begin{array}{l}\text { Urine volume } \\
(\mathrm{ml} / 10 \mathrm{~g} \mathrm{BW} / \mathrm{d})\end{array}$ & $0.78 \pm 0.03$ & $0.49 \pm 0.06^{\mathrm{A}}$ & $3.14 \pm 0.38$ & $1.44 \pm 0.26^{\mathrm{B}}$ \\
$\begin{array}{l}\text { Salt intake } \\
(\mu \mathrm{g} / \mathrm{g} \mathrm{BW} / \mathrm{d})\end{array}$ & $1.59 \pm 0.29$ & $1.22 \pm 0.30$ & $5.1 \pm 0.75$ & $1.28 \pm 0.80^{\mathrm{B}}$ \\
& & & &
\end{tabular}

Pre-losartan, before losartan administration; post-losartan, following losartan administration. ${ }^{A} P<0.05,{ }^{B} P<0.01$ versus before losartan administration.

(data not shown). Interestingly, this was the only region where an obvious increase in Ang II immunostaining was evident. This observation lead us to hypothesize that the increase in drinking was due to increased de novo production of Ang II in the SFO.

To directly test this hypothesis and to distinguish de novo synthesis of Ang II in the SFO from synthesis of circulating or ventricular Ang II detected by the SFO, we examined the drinking response after targeted ablation of Ang II substrate (AGT) synthesis in the SFO. We took advantage of a genetic tool that we believe to be novel in order to accomplish this goal. We employed what we believe is a new double-transgenic strain (SRA ${ }^{\text {flox }}$ ), which was bred from a synapsin- $h R E N$ mouse (same as that used to generate SRA mice) crossed with a transgenic mouse engineered to express a modified $h A G T$ containing loxP sites surrounding exon II ( $h A G T^{f l o x}$ ) (29-31). The presence of loxP sites surrounding the major coding exon of the gene provides a genetic means of ablating the exon in the presence of Cre recombinase (27). In the absence of Cre recombinase, the $h A G T^{f l o x}$ gene is fully functional and expresses $h A G T$. However, when Cre recombinase is expressed, the gene is functionally crippled because of the deletion of exon II (29). Expression of Cre recombinase was achieved by stereotaxic microinjection of AdCre directly into the $\operatorname{SFO}(27,31)$.

We previously reported that the $h A G T^{\text {flox }}$ and $h A G T$ transgenes exhibit the same tissue-specific and cell-specific pattern of expression $(29,30)$. However, the level of $h A G T$ expression is higher in the brain of $h A G T^{f l o x}$ mice than in the brain of $h A G T$ mice (Figure $4 \mathrm{~A}$ ). An initial evaluation of 23 randomly chosen $S R A^{\text {flox }}$ mice revealed a marked increase in water intake averaging $6.8 \pm 0.6 \mathrm{ml} / 10 \mathrm{~g} \mathrm{BW} / \mathrm{d}$ and a concomitant increase in urine volume $(6.7 \pm 0.8 \mathrm{ml} / 10 \mathrm{gBW} / \mathrm{d})$ and salt intake $(5.7 \pm 0.5 \mu \mathrm{g} / \mathrm{g} \mathrm{BW} / \mathrm{d})$. Although the magnitude of the drinking response was less, a highly significant increase in water intake $(P<0.001)$ was also observed in a second independent study pairing SRA $A^{\text {flox }}$ mice with age- and sex-matched littermate controls (Figure 4B). SRA flox mice also exhibited an increase in angiotensin peptides in the SFO (Figure 3C).

Next, we directly targeted the SFO by microinjection of either adenovirus encoding EGFP (AdEGFP) alone or both AdEGFP and $A d C r e$ together. Seven days after the microinjection, site-specific expression of EGFP on brain sections was confirmed by examination with a fluorescent stereomicroscope (Figure 5A). To confirm Cre-mediated recombination, we obtained punches of brain tissue from EGFP-positive and -negative brain segments and performed RT-PCR to confirm the presence of either the normal $h A G T$ mRNA encoding exons $1-5$ or the Cre-deleted $h A G T$ mRNA lacking exon 2 (direct splicing of exons 1 to 3 , as previously reported in ref. 29).
Cre-mediated recombination was evident in those segments exhibiting EGFP staining but not in control segments (Figure 5B). At the cellular level, we confirmed that the level of hAGT protein in the SFO was unaltered in mice receiving only AdEGFP and that hAGT and EGFP were clearly coexpressed in the same cells (Figure 6, A-C). A few cells neighboring the SFO were also observed to express EGFP but not hAGT (arrows in Figure $6 \mathrm{C}$ ), indicating transduction of cells both in and just outside the SFO. On the contrary, there was a marked reduction in hAGT staining in the SFO of mice receiving both $A d E G F P$ and $A d C r e$, specifically in those cells expressing the EGFP reporter (Figure 6, D-F). Note that there was no overlap in the staining of hAGT and EGFP (the marker of Cre recombinase) except for perhaps 1 or 2 cells (arrow in Figure 6F).

We next measured water intake before and after injection of $A d C r e$. In the control groups, there was no effect on drinking in response to $A d E G F P$ injection into $\mathrm{SRA}^{\text {flox }}$ mice or $A d C r e / A d E G F P$ injection into SRA mice lacking $h A G T^{\text {flox }}$ (Figure $7 A$ ). There was also no decrease in water intake in SRA flox mice in which we confirmed that $A d C r e$ and $A d E G F P$ missed the SFO. On the contrary, AdEGFP/AdCre-transfected SRA ${ }^{\text {flox }}$ mice in which the SFO-specific microinjection was confirmed by EGFP fluorescence and RT-PCR exhibited a significant decrease in water intake that reached a nadir 2 days after injection and remained low for the next 6 days $(P<0.05)$ (Figure 7A). A comparison of the change in water intake over the 8-day period after AdCre/AdEGFP injection into SRA flox mice that either missed or hit the SFO clearly demonstrates that local hAGT production in the SFO was required to mediate the elevated drinking (Figure 7B).

Finally, to confirm that the decrease in drinking was due to decreased angiotensin peptides in the SFO, we performed immunocytochemistry for angiotensin I/II in the SFO of SRA flox mice that were either injected with AdEGFP alone (Figure 8A) or with AdCre/AdEGFP (Figure 8B). These data clearly show a significant decrease in angiotensin peptide staining after AdCre injection that matches the background of the immunocytochemical assay evaluated by eliminating the primary antibody (Figure 8C). There was no decrease in angiotensin peptide in the SFO when the injection of $A d C r e / A d E G F P$ was confirmed to have missed the SFO
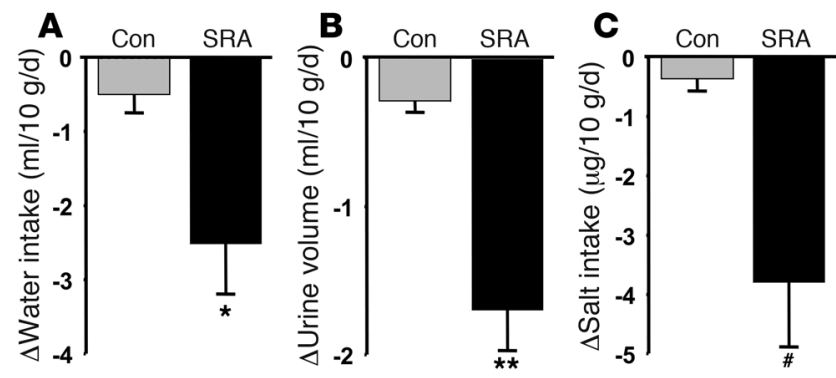

\section{Figure 2}

The effect of chronic icv administration of losartan on volume homeostasis in SRA mice. Change in water intake (A), urine volume (B), and salt intake (C) in SRA mice $(n=9)$ and nontransgenic mice $(n=6)$ in response to losartan administered icv. ${ }^{*} P=0.007(\mathbf{A})$; ${ }^{* \star} P<0.001$ (B); $\# P=0.002(\mathbf{C})$ compared with control mice. 

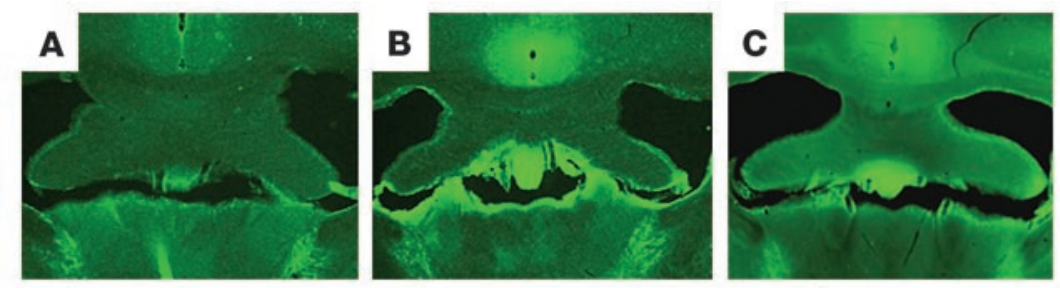

\section{Figure 3}

Localization of angiotensin peptide in the brain of SRA and SRA flox mice. Representative photomicrographs of immunostaining for Ang I/II peptide in the SFO by immunofluorescence $(\mathbf{A}-\mathbf{C})$ and immunoperoxidase (D and $\mathbf{E}$ ) in nontransgenic control ( $\mathbf{A}$ and $\mathbf{D})$, SRA (B and $\mathbf{E}$ ), and SRA ${ }^{\text {flox }}$ (C) mice. $n=4$ or more mice. Scale bars: $0.4 \mathrm{~mm}(\mathbf{A}-\mathbf{C}) ; 0.1 \mathrm{~mm}$ (D and $\mathbf{E})$.

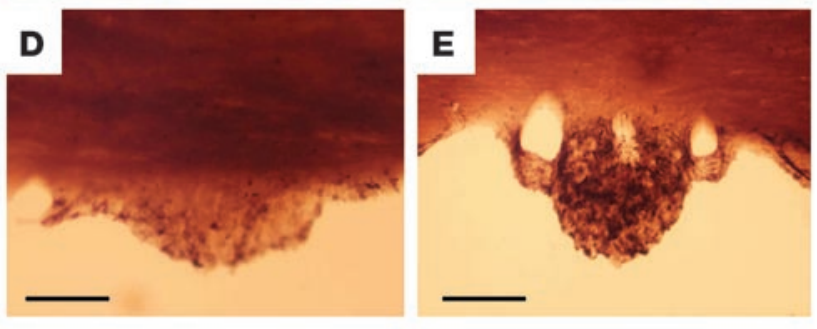

SRA and SRA ${ }^{\text {flox }}$ models employed herein to assess the physiological consequences of elevated de novo Ang II production under conditions in which renin and AGT were correctly expressed in a cell-specific manner. In our model, the synapsin I promoter targeted expression of renin to neurons, while the endogenous $A G T$ promoter targeted its expression to glial cells and, importantly, the correct population of $A G T$-expressing neurons (22).

(Figure 8D). Ultrastructural analysis showed strong angiotensin peptide staining in an SFO neuron in the nucleus and cytosol and vesicular structures of uninjected SRA ${ }^{\text {flox }}$ mice (Figure 9, A and B) but a significant decrease in electron-dense material after injection of AdCre/AdEGFP (Figure 9C).

\section{Discussion}

There is now considerable evidence that all components of the RAS exist within the brain. At the cellular level, angiotensin II is located in neuronal somata and axons and at nerve terminals (14). Angiotensin receptors are abundantly expressed on postsynaptic membranes of cell bodies as well as on neuronal fibers $(34,35)$. The location of Ang II immunoreactive fibers as well as binding sites for Ang II correlate well with nuclei controlling blood pressure, water and electrolyte homeostasis, and baroreceptor reflex pathways (36). Exogenous administration of Ang II into Ang II receptor-containing regions of the brain elicits both blood pressure and drinking responses, and the blockade of endogenous Ang II production or action has effects on both (reviewed in ref. 37). Although the central actions of exogenous Ang II are well documented, the mechanisms controlling its de novo production in the brain remain very incomplete.

The Ang II substrate, AGT, is abundantly expressed and widely distributed in astroglia (8). Other reports suggest that AGT is also expressed in neurons, although in a much more regionally restricted manner $(7,22)$. Renin, on the other hand, has been difficult to identify because its level of expression is quite low and commonly available antisera directed at renin are only marginally effective for immunohistochemical methods. Using a pair of transgenic mice expressing sensitive reporter genes under the control of the renin and $A G T$ promoters, we recently identified regions of the brain containing renin-expressing neurons and AGT-expressing cells that were both closely localized (adjacent) and colocalized (expressed in the same cell) (13). We therefore developed the

\section{Figure 4}

Expression hAGT in the brain and water intake in SRA ${ }^{\text {flox }}$ mice. (A) An RNase protection assay of total RNA purified from independent brain samples removed from 3 SRA and 3 SRA flox mice. The positions of the $h A G T$ - and 28S-protected bands are shown. (B) Water intake in $\operatorname{SRA}^{\text {flox }}(n=4)$ and age- and sex-matched littermate controls $(n=9)$. ${ }^{\star} P<0.001$ compared with control mice.
This cell-appropriate overexpression of Ang II resulted in a striking increase in water intake, salt preference, and urine output. Immunostaining revealed that the SFO was the only nucleus with an obvious increase in immunoreactive angiotensin peptides. Interestingly, inspection of multiple brain sections did not provide convincing evidence for elevated Ang II production in other nuclei implicated in drinking, such as the median preoptic nucleus $(\mathrm{MnPO})$ or the paraventricular nucleus (PVN). Consequently, it is possible that in this model system, the SFO was the only nucleus where hREN and hAGT were sufficiently closely localized to generate Ang II. Because the promoter elements used to target expression of the $h R E N$ and $h A G T$ genes emulated their natural cellular pattern of expression, one might hypothesize that the SFO is one of the primary locations for de novo synthesis of Ang II in the brain. Nevertheless, future studies will have to address whether enhanced production of Ang II, perhaps at a lower level than is easily detected by immunocytochemistry, occurred in other nuclei regulating fluid homeostasis. Similarly, as our experiment is admittedly an overexpression study, future studies must address whether local production of Ang II in the SFO is required to mediate drinking responses under normal physiological conditions.

The SFO has long been thought to be a critical integrating center for regulating many of the physiological responses of Ang II, including drinking (19). Neurons in the SFO are rich in $\mathrm{AT}_{1}$ receptors, and Ang II immunoreactive fibers can be traced from the SFO to other regions implicated in homeostatic control (24). Destruction of these pathways by lesioning the $\mathrm{SFO}$ or regions such as the $\mathrm{MnPO}$, which contain angiotensinergic projections from the SFO, or transection

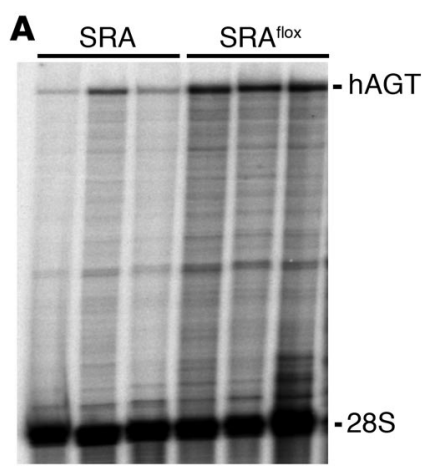

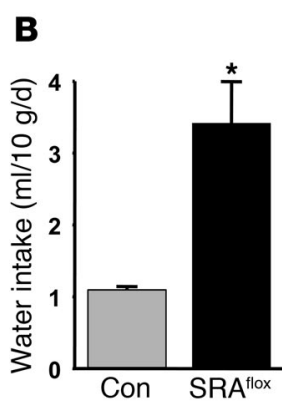



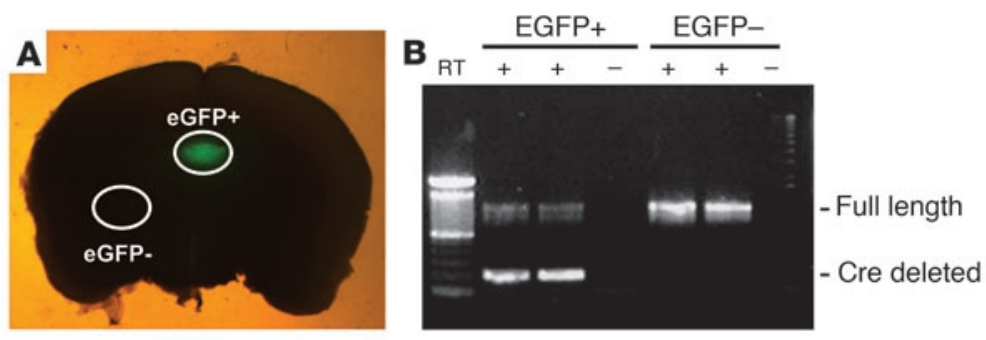

Figure 5

Site-specific deletion of $h A G T$ mRNA in the SFO. (A) Representative brain slice from an $S_{R A} A^{\text {flox }}$ mouse coinfected with AdEGFP and AdCre and photographed under a fluorescence stereomicroscope. Selected from over 12 photos each from independent mice $(n=12)$. Brain tissue from an EGFP-positive region and from a control region (EGFP-) were carefully dissected for the experiment in B. (B) RT-PCR reaction for $h A G T$ mRNA from brain punches derived from the EGFP-positive and -negative regions indicated in $\mathbf{A}$.

of these neural pathways abolishes the drinking response to i.v. or locally administered Ang II $(24,25,38)$. That elevated drinking in the SRA model was reduced to baseline when losartan was delivered chronically into the brain strongly supports the dipsogenic response as being $\mathrm{AT}_{1}$ mediated. Our previous studies suggest the $\mathrm{AT}_{1 \mathrm{~B}}$ subtype of $\mathrm{AT}_{1}$ receptors may mediate the dipsogenic responses to Ang II (39). Although the drinking response was completely normalized by $\mathrm{AT}_{1}$ receptor blockade, future experiments will be required to assess the relative contribution of $\mathrm{AT}_{1 \mathrm{~A}}$ and $\mathrm{AT}_{1 \mathrm{~B}}$ receptors or the role, if any, of $\mathrm{AT}_{2}$ receptors, which have also been implicated in regulating the drinking response to central Ang II (40).

The SFO is among a number of circumventricular organs that have access to the circulation. Several models have been proposed to explain the mechanism of angiotensinergic stimulation of the SFO (reviewed in ref. 41). In one model, an SFO neuron takes up circulating Ang II by a receptor-mediated mechanism and then releases Ang II inside the cell. The internalized Ang II then undergoes axonal transport and is ultimately released as a neurotransmitter at the synapse, where it binds to $\mathrm{AT}_{1}$ receptors on the postsynaptic membrane of the connecting neuron or interneuron. Despite the implication that Ang II acts as a neurotransmitter between the SFO and its efferent targets, the one criterion that has long been lacking to classify Ang II as a neurotransmitter is de novo synthesis of Ang II in the presynaptic neuron (reviewed in ref. 41). It is interesting to note therefore that the SFO is one of the sites in the central nervous system containing both neuronal AGT and renin, which suggests the potential for de novo local synthesis of Ang II even when circulating levels of Ang II are normal $(13,22)$. Our data showing that ablation of Ang II substrate synthesis specifically in the SFO markedly diminishes Ang II production in the SFO and attenuates drinking provides compelling evidence for the local production of Ang II in the SFO and its role in signaling the dipsogenic response. The data also complement our previous studies demonstrating that ablation of SFO AGT attenuates the pressor response to an icv infusion of recombinant hREN (31). These data therefore provide an additional criterion implicating Ang II as a neurotransmitter originating from neuron cell bodies located in the SFO. Our data also suggest the possibility that there are "microdomains" of Ang II synthesis in critical regions of the CNS controlling Ang II-dependent neural output and that Ang II production in these microdomains may be under tight physiological regulation. One of these regions, the rostral ventral lateral medulla (RVLM), controls sympathetic outflow and expresses renin and AGT locally (13). A recent, elegant study by Allen et al. showed that targeting a constitutively active $\mathrm{AT}_{1}$ receptor to glial cells in the RVLM caused hypertension (42). One model posited by the authors is that glial $\mathrm{AT}_{1}$ receptors may modulate the synthesis, and perhaps release, of glial AGT, thus providing a regulatory circuit controlling the level of Ang II substrate available to interact with neuronal renin.

In interpreting our results, we recognize that the level of drinking was not completely normalized after SFO-specific delivery of Cre recombinase. This would be consistent with either an incomplete ablation of Ang II production in the SFO or local Ang II synthesis in other hypothalamic nuclei controlling drinking (43). Whereas our immunocytochemical data show efficient ablation of hAGT synthesis in EGFP-labeled cells, the extent of EGFP reporter expression in the SFO varied among experimental mice. Consequently, the most likely explanation is incomplete ablation of Ang II production in the SFO. However, although we did not obtain convincing evidence for elevated Ang II production in other nuclei

\section{Figure 6}

Site-specific deletion of hAGT protein in the SFO. Representative coronal sections through the SFO from mice injected with either AdEGFP alone or both AdEGFP and $A d C r e$ directly into the SFO. AGT was localized by immunofluorescence (red) and EGFP by its intrinsic fluorescence (green). A-C show colocalization of AdEGFP and AGT but no deletion or diminution of AGT staining. The arrows in $\mathbf{C}$ indicate EGFP-positive, hAGT-negative cells adjacent to the SFO. D-F show loss of AGT in regions where the virus mixture (AdEGFP and $A d C r e$ ) is located. The arrow in $\mathbf{F}$ represents a cell in which hAGT was not ablated, which may represent a cell infected with AdEGFP but not AdCre.
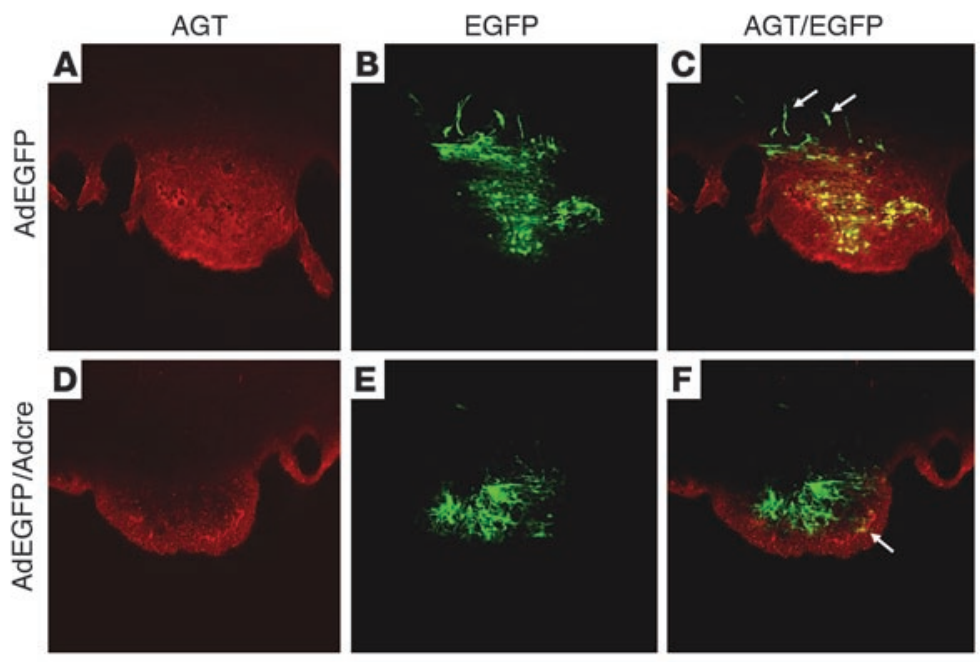

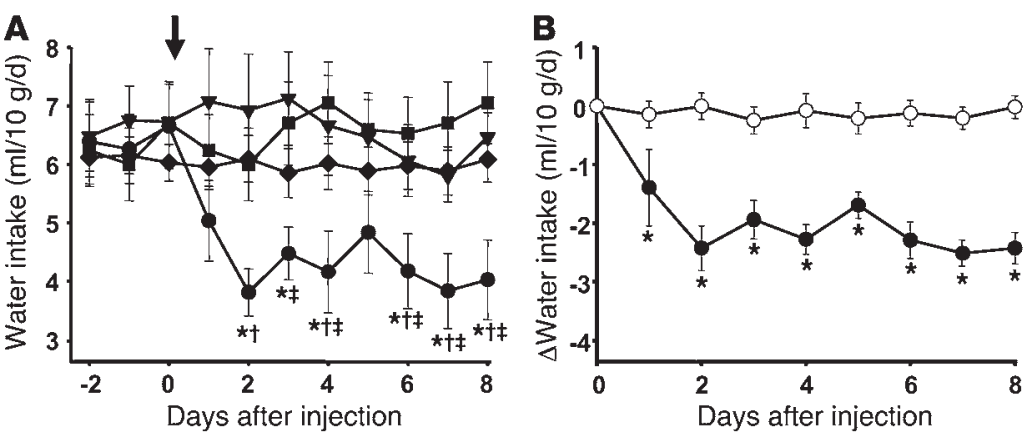

Figure 7

Effect of AdCre transfection into the SFO on water intake. (A) Drinking volume of water before and after SFO-directed injection (arrow) of AdCre/AdEGFP into SRA mice lacking loxP sites (filled squares; $n=8$ ); AdEGFP alone into $S_{R} A^{\text {flox }}$ mice (filled triangles; $n=8$ ); AdCre/AdEGFP into SRA flox mice, where the injection missed the SFO (filled diamonds; $n=21$ ); and AdCre/AdEGFP into SRA ${ }^{\text {flox }}$ mice, where the injection into the SFO was confirmed (filled circles; $n=8$ ). Data were analyzed using 2-way repeated measures ANOVA with the Bonferroni posthoc test. ${ }^{*} P<0.05$ versus SRA flox mice injected with $A d E G F P ;{ }^{\dagger} P<0.05$ versus $S_{R A} A^{\text {flox }}$ mice injected with AdCre, which missed the SFO; $¥ P<0.05$ versus SRA mice lacking loxP sites injected with AdCre/AdEGFP. (B) Change in water intake by mice after SFO-directed injection of AdCre/AdEGFP into SRA flox mice, where the injection missed the SFO (open circles) and of AdCre/AdEGFP into SRA flox mice, where the injection into the SFO was confirmed (filled circles). ${ }^{*} P<0.003$.

implicated in drinking, the importance of these nuclei cannot be ruled out by the studies performed herein. Consequently, studies must be undertaken to determine whether de novo Ang II production in other circumventricular organs or in nuclei along the neural pathway initiated in the SFO is necessary for regulating angiotensinergic dipsogenic responses initiated in the SFO. Whereas our results indicate that de novo synthesis of Ang II in the SFO is necessary for increased water intake, studies are currently underway to assess whether Ang II production in the SFO and elsewhere is sufficient to mediate increased water intake.

Along these lines, we and others have provided convincing evidence that the Cre-loxP recombinase system offers a unique tool to dissect neural pathways in the brain (31). At the level of the whole brain, cell-specific promoters can be used to target Cre recombinase expression to specific cell types (i.e., neurons or glia). Although our ability to target specific populations of neurons has improved (44, 45), examining regional angiotensinergic pathways has become more difficult, as promoters that have the ability to target specific regions of the brain, such as the SFO or MnPO, have yet to be identified. Certainly will be one of the long-anticipated outcomes of the brain genome and mapping projects (46). In the meantime, viral delivery of Cre recombinase provides an innovative tool for the region-specific ablation or even activation of gene expression (47). Recent technological developments that employ retrograde transport of infected viruses or the cellular tropism of specific viruses have provided a means to target specific cell types in the brain, follow specific neuronal pathways, and ablate expression of genes at sites distant from the sites of infection $(27,48)$. Although we did not observe evidence for retrograde transport of AdEGFP from the SFO in our experimental system, studies could be performed in which the virus is injected into the $\mathrm{PVN}$ or $\mathrm{MnPO}$ to assess the relative contribution of the SFO-MnPO and SFO-PVN pathways. The next obvious step will be to use the molecular tools developed herein to dissect the neuronal pathways mediating the drinking response.

\section{Methods}

Animal models. Transgenic mice encoding the hREN gene under the control of neuron-specific promoter synapsin I (SR mice), and $b A G T$ under its endogenous promoter, were previously described $(28,32)$. We also previously generated and characterized transgenic mice containing the $b A G T$ gene in which exon II, the major coding exon of the gene, is flanked by loxP sites (termed $h A G T^{f l o x}$ ) and an AdCre $(29,30)$. All mice were maintained by successive generations of backcross breeding to C57BL/6J mice (The Jackson Laboratory). SRA and SRA ${ }^{\text {flox }}$ double-transgenic mice were generated by crossbreeding heterozygous SR mice with heterozygous $h A G T$ or $h A G T^{f l o x}$ transgenic mice. Double-transgenic mice were identified by PCR of tail DNA using $h R E N$ - and $h A G T$-specific primers as described in refs. 28 and 32. Because of the strong species specificity of the renin-AGT enzymatic reaction, there is no significant phenotypic difference between single-transgenic $S R^{+} / A^{-}$or $S R^{-} / A^{+}$and nontransgenic $S R^{-} / A^{-}$animals (49). Therefore, only 2 experimental groups (SRA and SRA ${ }^{\text {flox }}$ ) and 1 control group (single-transgenic or nontransgenic littermates) were used in our studies. All mice were fed standard mouse chow (LM-485; Teklad Premier Laboratory Diets) and water ad libitum. Care of the mice used in the experiments met the standards set forth by the NIH in their guidelines for the care and use of experimental animals. All procedures were approved by the University Animal Care and Use Committee at the University of Iowa.

Viruses. Adenoviruses were constructed in the Gene Transfer Vector Core at the University of Iowa. We used 2 adenoviral vectors, AdEGFP and AdCre. We microinjected either AdEGFP alone (as a control) or a mixture of AdCre and AdEGFP into the SFO of SRA ${ }^{\text {flox }}$ and SRA mice (27). Mice 16-20 weeks of age were anesthetized and placed in a stereotaxic frame, and a hole was made $0.65 \mathrm{~mm}$ posterior to the bregma. A glass micropipette ( $5 \mu \mathrm{m} \mathrm{OD}$ ) was filled with the viruses and inserted $2.9 \mathrm{~mm}$ below the skull surface. The site of microinjection was defined according to an atlas of the mouse brain (50). The adenoviral suspension contained $5 \times 10^{9} \mathrm{PFU} / \mathrm{ml}$ and was injected at a rate of
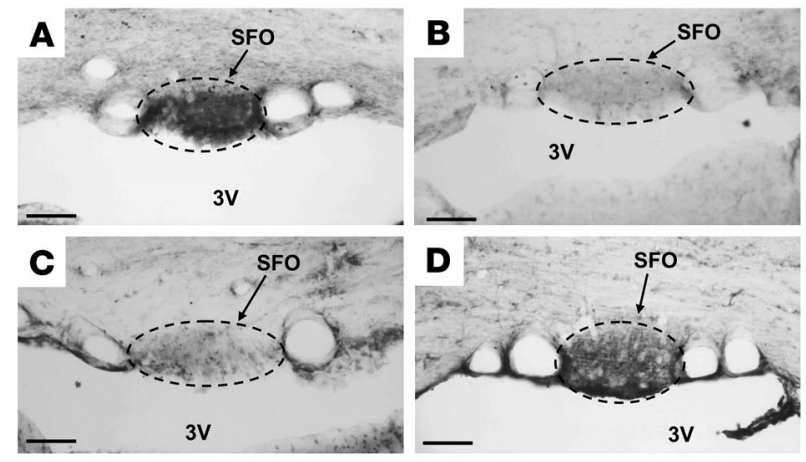

\section{Figure 8}

Effect of AdCre on SFO Ang II. Immunocytochemistry for angiotensin $I / I I$ in the SFO of SRA flox mice. (A) SRA flox mouse injected with AdEGFP alone. (B) Diminished Ang I/II signal in SFO after SFO-directed injection of AdCre/AdEGFP. (C) Control SFO section from SRA flox mice where the primary antibody directed against Ang I/II was omitted. (D) Retention of Ang I/II immunoreactivity in SFO after an injection of AdCre/AdEGFP that missed the SFO. The SFO is bordered by a dashed line. $3 \mathrm{~V}$, third ventricle. Scale bars: $100 \mu \mathrm{m}$. 

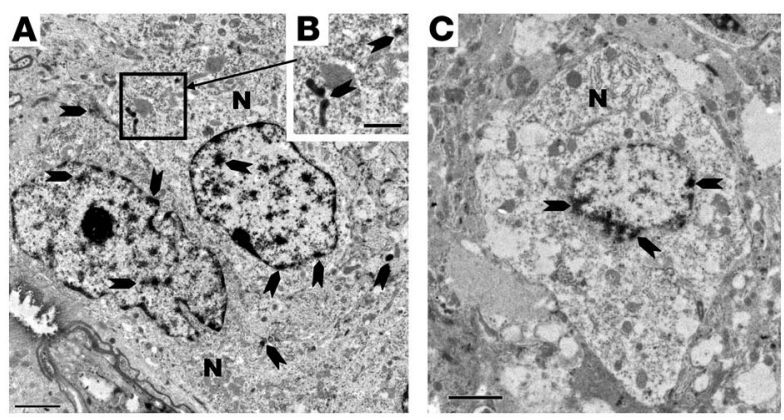

Figure 9

Ultrastructural localization of Ang I/II in the SFO. (A) Strong expression of Ang I/II (arrows) in the nucleus, cytosol, and vesicular structures of SFO neurons of an uninjected SRA ${ }^{\text {flox }}$ mouse. (B) Fragment of cytoplasm of an SFO neuron in A containing immunoreactive vesicular structures (arrows). (C) Note the very low level of immunoreactivity around the neuronal nucleus (arrows) and absence of immunoreactivity in cytoplasm and neuropil after AdCre/AdEGFP was directly injected into the SFO. N, neuron. Scale bars: $2.0 \mu \mathrm{m}$ (A and C); $1.0 \mu \mathrm{m}$ (B).

$0.4 \mu \mathrm{l} / \mathrm{min}$ (100 $\mathrm{nl}$ total volume). After the injection, all mice were allowed to recover from anesthesia and were free to move around their home cage.

Physiological measurements. Water and salt intake were measured as described previously (28). Briefly, water intake of individual mice in metabolic cages with standard chow and tap water in burettes ad libitum was measured once per day. To determine salt intake, mice were fed salt-deficient chow and given $0.3 \mathrm{~mol} / \mathrm{l}$ hypertonic saline and tap water in separate, randomized burettes. Salt intake was calculated as a total weight of salt that each mouse consumed in 24 hours. Urinary volume was measured by collecting 24-hour urine in metabolic cages with standard chow ground and mixed with tap water (100 g chow/150 $\mathrm{ml}$ water) and tap water ad libitum daily. Baseline measurements were obtained individually and were calculated as the mean during 3 consecutive days.

Some mice were also instrumented with icv cannulas for chronic microinfusion of either $\mathrm{AT}_{1}$ receptor antagonist, losartan, or artificial CSF into the lateral ventricle as previously described $(31,33,39)$. Briefly, mice were placed in a stereotaxic apparatus so that the head was aligned and the lambda-bregma plane was horizontal. A hole was made $0.3 \mathrm{~mm}$ posterior and $1.0 \mathrm{~mm}$ lateral to the bregma, and a cannula (25 gauge) was inserted $3.2 \mathrm{~mm}$ below the skull surface and fixed in place. Drug infusions were made through the cannula, which was connected to PE-50 tubing fitted with osmotic minipumps (model 1002, Alzet; DURECT Corp.) implanted subcutaneously. $\mathrm{AT}_{1}$ receptor antagonist, losartan $(1 \mu \mathrm{g} / 0.21 \mu \mathrm{l} / \mathrm{h})$, dissolved in aCSF (in mmol/l: NaCl 136, KCl 5.6, $\mathrm{NaHCO}_{3}$ 16.2, $\mathrm{NaH}_{2} \mathrm{PO}_{3}$ $1.2, \mathrm{MgCl}_{2} 1.2$, and $\mathrm{CaCl}_{2} 2.2$, $\mathrm{pH}$ 7.4), was infused chronically for 14 days. Chronic icv infusion of $\mathrm{aCSF}(0.21 \mu \mathrm{l} / \mathrm{h})$ or chronic subcutaneous infusion of losartan $(1 \mu \mathrm{g} / 0.21 \mu \mathrm{l} / \mathrm{h})$ was performed as a control. Water, salt intake, and urinary volume were calculated for 4 consecutive days (days 8 to 11) after the icv administration of losartan. The same measurements were recorded for 8 consecutive days after microinjection of adenoviruses.

Molecular assays. Brains from control, SRA, and SRA flox mice were harvested, snap-frozen in liquid nitrogen, and RNA was purified using TriReagent (Molecular Research Center Inc.). We confirmed the local expression of Cre recombinase produced by $\mathrm{AdCre}$ in and near the SFO by using a fluorescent stereomicroscope to identify the region labeled by EGFP. A 1-mm-thick coronal brain section that included the SFO was cut with a brain matrix (catalog number BS-5000C; Braintree Scientific) and quickly frozen. A section containing SFO transfected with AdCre or AdEGFP was placed on a glass dish and photographed under a fluorescent stereomicroscope with a 488-nm excitation beam and appropriate filter sets to visualize EGFP expression. We then excised the fluorescent material by needle punch. We performed RT-PCR on RNA derived from this excised tissue to confirm Cre-mediated gene deletion. A 1,060-bp fragment for the non-recombined DNA fragment and 202-bp fragment for recombined DNA fragment were expected using the following primers: 5'-GCTGCCGTTGTTCTGGGTA-3' and 5'-GCAGGCAGGCGCTCTCAGT-3' $(29,30)$.

$A G T$ and Ang I/II immunocytochemistry. Immunocytochemistry for hAGT was performed as previously described $(28,51)$. For angiotensin peptides, nontransgenic, SRA, and SRA flox mice were anesthetized and perfused with PBS containing 4\% paraformaldehyde and $1.0 \%$ glutaraldehyde. Brains were removed, postfixed in the same fixative for 24 hours and cut by vibratome into 35- to $50-\mu \mathrm{m}$-thick coronal sections. Sections were then incubated overnight in a 1:300 dilution of primary goat anti-Ang I/II antibody (N-10 Angiotensin I/II antisera, catalog number sc-7419; Santa Cruz Biotechnology Inc.). Sections were then washed with PBS and placed in 1:200 biotinylated secondary antibody, followed by biotin detection with solutions from VECTASTAIN ABC Elite kit (Vector Laboratory). Visualization was with treatment with 3,3'-diaminobenzidine (DAB) plus hydrogen peroxide or FITC-labeled streptavidin. For control studies, the adjacent sections were either processed using the same protocol but without primary antibody or processed with primary antisera that was preabsorbed with immunizing peptide. Preabsorption eliminated Ang I/II immunoreactivity in the SFO from both nontransgenic and SRA ${ }^{\text {flox }}$ mice. Sections were coverslipped and observed with a Nikon light microscope. Sections taken for electron microscopic analysis were postfixed with $1 \% \mathrm{OsO}_{4}$, rinsed, dehydrated, and flat embedded in a mixture of Epon-812 and Araldite-502 (both from Electron Microscopic Sciences). The SFO was photographed using a Nikon microscope, then cut out from flat section using beveled cuts to preserve orientation. Ultrathin sections were cut using a Leica ultramicrotome, after which the sections were stained with uranyl acetate and lead citrate and examined under a Jeol-1230 electron microscope. Under electron microscopic examination, we considered a structure to be labeled (immunoreactive) if it showed a highly electron-dense $\mathrm{DAB}$ reaction product in comparison with that shown in adjacent structures.

Digital imaging. All light microscopic images were taken with a digital camera (Sciscope Instrument Co.) attached to a Nikon light microscope. Electron microscopic images were also taken with a Gatan UltraScan 1000 $2 \mathrm{k} \times 2 \mathrm{k}$ charge-coupled device digital camera.

Statistics. All values are expressed as mean \pm SEM. Data were analyzed by 2 -tailed Student's $t$ test when only 2 groups were compared. For the 4-group analysis of the drinking response to SFO-specific injection of AdCre, we used 2-way repeated measures ANOVA with the Bonferroni posthoc correction for multiple comparisons. All data were analyzed with SigmaStat (version 3.5; Systat Software). Differences were considered significant at $P<0.05$.

\section{Acknowledgments}

This work was supported by grants from the NIH (HL48058, HL61446, and HL55006 to C.D. Sigmund; HL63887 and HL14388 to R.L. Davisson), the American Heart Association (0030017N and 0540114 N to R.L. Davisson). K. Sakai was previously funded by the Japan Society for the Promotion of Science. P. Sinnayah was funded by a postdoctoral fellowship from the American Heart Association (0225723Z). K. Agassandian was funded by the Center on Functional Genomics of Hypertension. Transgenic mice were generated and maintained at the University of Iowa Transgenic Animal Facility, supported by the Roy J. and Lucille A. Carver College of Medicine. AdCre and AdEGFP viruses were obtained from the University of Iowa Vector Core, DNA sequencing was performed at the University of Iowa DNA Core, and the dissecting fluorescence 
microscope was used in the Central Microscopy Facility of the University of Iowa. We gratefully acknowledge the generous research support of the Roy J. Carver Charitable Trust.

Received for publication December 15, 2006, and accepted in revised form February 20, 2007.

1. Johnson, A.K., and Epstein, A.N. 1975. The cerebral ventricles as the avenue for the dipsogenic action of intracranial angiotensin. Brain Res. 86:399-418.

2. Buggy, J., Fisher, A.E., Hoffman, W.E., Johnson, A.L., and Phillips, M.I. 1975. Ventricular obstruction: effect on drinking induced by intracranial injection of angiotensin. Science. 190:72-74.

3. Buggy, J., and Fisher, A.E. 1974. Evidence for a dual central role for angiotensin in water and sodium intake. Nature. 250:733-735.

4. Phillips, M.I., et al. 1977. Lowering of hypertension by central saralasin in the absence of plasma renin. Nature. 270:445-447.

5. DiNicolantonio, R. 1984. Angiotensin converting enzyme blockade and thirst. Clin. Exp. Hypertens. A. 6:2025-2029.

6. Epstein, A.N., Fitzsimons, J.T., and Johnson, A.K. 1973. Prevention by angiotensin II antiserum of drinking induced by intracranial angiotensin. J. Physiol. 230:42P-43P.

7. Thomas, W.G., and Sernia, C. 1988. Immunocytochemical localization of angiotensinogen in the rat brain. Neuroscience. 25:319-341.

8. Stornetta, R.L., Hawelu-Johnson, C.L., Guyenet, P.G., and Lynch, K.R. 1988. Astrocytes synthesize angiotensinogen in brain. Science. 242:1444-1446.

9. Imboden, H., Harding, J.W., Hilgenfeldt, U., Celio, M.R., and Felix, D. 1987. Localization of angiotensinogen in multiple cell types of rat brain. Brain Res. 410:74-77.

10. Fuxe, K., et al. 1980. Renin-like immunocytochemical activity in the rat and mouse brain. Newrosci. Lett. 18:245-250

11. Slater, E.E., Defendini, R., and Zimmerman, E. 1980. Wide distribution of immunoreactive renin in nerve cells of human brain. Proc. Natl. Acad. Sci. U. S. A. 77:5458-5460.

12. Lavoie, J.L., Cassell, M.D., Gross, K.W., and Sigmund, C.D. 2004. Localization of renin expressing cells in the brain using a REN-eGFP transgenic model. Physiol. Genomics. 16:240-246.

13. Lavoie, J.L., Cassell, M.D., Gross, K.W., and Sigmund, C.D. 2004. Adjacent expression of renin and angiotensinogen in the rostral ventrolateral medulla using a dual-reporter transgenic model. Hypertension. 43:1116-1119.

14. Lind, R.W., Swanson, L.W., and Ganten, D. 1985. Organization of angiotensin II immunoreactive cells and fibers in the rat central nervous system. An immunohistochemical study. Neuroendocrinology. 40:2-24.

15. Sinn, P.L., and Sigmund, C.D. 2000. Identification of three human renin $\mathrm{mRNA}$ isoforms resulting from alternative tissue-specific transcriptional initiation. Physiol. Genomics. 3:25-31.

16. Re, R.N. 2003. Intracellular renin and the nature of intracrine enzymes. Hypertension. 42:117-122.

17. Lee-Kirsch, M.A., Gaudet, F., Cardoso, M.C., and Lindpaintner, K. 1999. Distinct renin isoforms generated by tissue-specific transcription initiation and alternative splicing. Circ. Res. 84:240-246.

18. Pickel, V.M., Chan, J., and Ganten, D. 1986. Dual peroxidase and colloidal gold-labeling study of angiotensin converting enzyme and angiotensinlike immunoreactivity in the rat subfornical organ. J. Neurosci. 6:2457-2469.
Address correspondence to: Curt D. Sigmund, Departments of Internal Medicine and Molecular Physiology and Biophysics, 3181B Medical Education and Biomedical Research Facility, Roy J. and Lucille A. Carver College of Medicine, University of Iowa, Iowa City, Iowa 52242, USA. Phone: (319) 335-7604; Fax: (319) 353-5350; E-mail: curt-sigmund@uiowa.edu.
19. Simpson, J.B., and Routtenberg, A. 1973. Subfornical organ: site of drinking elicitation by angiotensin II. Science. 181:1172-1175.

20. Fitzsimons, J.T. 1998. Angiotensin, thirst, and sodium appetite. Physiol. Rev. 78:583-686.

21. Simpson, J.B., and Routtenberg, A. 1978. Subfornical organ: a dipsogenic site of action of angiotensin II. Science. 201:379-381.

22. Yang, G., Gray, T.S., Sigmund, C.D., and Cassell, M.D. 1999. The angiotensinogen gene is expressed in both astrocytes and neurons in murine central nervous system. Brain Res. 817:123-131.

23. Hoffman, W.E., and Phillips, M.I. 1976. The effect of subfornical organ lesions and ventricular blockade on drinking induced by angiotensin II. Brain Res. 108:59-73.

24. Eng, R., and Miselis, R.R. 1981. Polydipsia and abolition of angiotensin-induced drinking after transections of subfornical organ efferent projections in the rat. Brain Res. 225:200-206.

25. Tanaka, J., and Nomura, M. 1993. Involvement of neurons sensitive to angiotensin II in the median preoptic nucleus in the drinking response induced by angiotensin II activation of the subfornical organ in rats. Exp. Neurol. 119:235-239.

26. Lavoie, J.L., and Sigmund, C.D. 2003. Minireview: overview of the renin-angiotensin system-an endocrine and paracrine system. Endocrinology. 144:2179-2183.

27. Sinnayah, P., et al. 2004. Targeted viral delivery of Cre recombinase induces conditional gene deletion in cardiovascular circuits of the mouse brain. Physiol. Genomics. 18:25-32.

28. Morimoto, S., Cassell, M.D., and Sigmund, C.D. 2002. Glial- and neuronal-specific expression of the renin-angiotensin system in brain alters blood pressure, water intake, and salt preference. J. Biol. Chem. 277:33235-33241.

29. Stec, D.E., Davisson, R.L., Haskell, R.E., Davidson, B.L., and Sigmund, C.D. 1999. Efficient liver-specific deletion of a floxed human angiotensinogen transgene by adenoviral delivery of cre-recombinase in vivo. J. Biol. Chem. 274:21285-21290.

30. Stec, D.E., Keen, H.L., and Sigmund, C.D. 2002. Lower blood pressure in floxed angiotensinogen mice after adenoviral delivery of cre-recombinase. Hypertension. 39:629-633.

31. Sinnayah, P., et al. 2006. Genetic ablation of angiotensinogen in the subfornical organ of the brain prevents the central angiotensinergic pressor response. Circ. Res. 99:1125-1131.

32. Yang, G., Merrill, D.C., Thompson, M.W., Robillard, J.E., and Sigmund, C.D. 1994. Functional expression of the human angiotensinogen gene in transgenic mice. J. Biol. Chem. 269:32497-32502.

33. Davisson, R.L., et al. 1998. The brain renin-angiotensin system contributes to the hypertension in mice containing both the human renin and human angiotensinogen transgenes. Circ. Res. 83:1047-1058.

34. Glass, M.J., Kruzich, P.J., Colago, E.E., Kreek, M.J., and Pickel, V.M. 2005. Increased AMPA GluR1 receptor subunit labeling on the plasma membrane of dendrites in the basolateral amygdala of rats selfadministering morphine. Synapse. 58:1-12.

35. Huang, J., et al. 2003. Angiotensin II subtype 1A (AT1A) receptors in the rat sensory vagal com- plex: subcellular localization and association with endogenous angiotensin. Neuroscience. 122:21-36.

36. Bunnemann, B., et al. 1992. The distribution of angiotensin II AT1 receptor subtype mRNA in the rat brain. Neurosci. Lett. 142:155-158.

37. Printz, M.P., Ganten, D., Unger, T., and Phillips, M.I. 1982. The brain renin-angiotensin system. In The renin-angiotensin system in the brain: a model for the synthesis of peptides in the brain. D. Ganten, M.P. Printz, M.I. Phillips, and B.A. Scholkens, editors. Springer-Verlag. Berlin, Germany. 3-52.

38. Simpson, J.B., Epstein, A.N., and Camardo, J.S., Jr. 1978. Localization of receptors for the dipsogenic action of angiotensin II in the subfornical organ of rat. J. Comp. Physiol. Psychol. 92:581-601.

39. Davisson, R.L., Oliverio, M.I., Coffman, T.M., and Sigmund, C.D. 2000. Divergent functions of angiotensin II receptor isoforms in brain. J. Clin. Invest. 106:103-106.

40. Camara, A.K., and Osborn, J. 2001. Central AT1 and AT2 receptors mediate chronic intracerebroventricular angiotensin II-induced drinking in rats fed high sodium chloride diet from weaning. Acta. Physiol. Scand. 171:195-201.

41. Ferguson, A.V., Washburn, D.L., and Latchford, K.J. 2001. Hormonal and neurotransmitter roles for angiotensin in the regulation of central autonomic function. Exp. Biol. Med. (Maywood). 226:85-96.

42. Allen, A.M., et al. 2006. Expression of constitutively active angiotensin receptors in the rostral ventrolateral medulla increases blood pressure. Hypertension. 47:1054-1061.

43. Tanaka, J., et al. 2001. Angiotensinergic and noradrenergic mechanisms in the hypothalamic paraventricular nucleus participate in the drinking response induced by activation of the subfornical organ in rats. Behav. Brain Res. 118:117-122.

44. Xu, A.W., Ste-Marie, L., Kaelin, C.B., and Barsh, G.S. 2007. Inactivation of signal transducer and activator of transcription 3 in proopiomelanocortin (Pomc) neurons causes decreased pomc expression, mild obesity, and defects in compensatory refeeding. Endocrinology. 148:72-80.

45. Kwon, C.H., et al. 2006. Neuron-specific enolasecre mouse line with cre activity in specific neuronal populations. Genesis. 44:130-135.

46. Gray, P.A., et al. 2004. Mouse brain organization revealed through direct genome-scale TF expression analysis. Science. 306:2255-2257.

47. Kilby, N.J., Snaith, M.R., and Murray, J.A.H. 1994. Site-specific recombinases: tools for genome engineering. Trends Genet. 9:413-421.

48. Sinnayah, P., et al. 2002. Selective gene transfer to key cardiovascular regions of the brain: comparison of two viral vector systems. Hypertension. 39:603-608.

49. Hatae, T., Takimoto, E., Murakami, K., and Fukamizu, A. 1994. Comparative studies on species-specific reactivity between renin and angiotensinogen. Mol. Cell. Biochem. 131:43-47.

50. Franklin, K.B.J., and Paxinos, G. 1997. The mouse brain in stereotaxic coordinates. Academic Press. San Diego, California, USA. 216 pp.

51. Morimoto, S., Cassell, M.D., and Sigmund, C.D. 2002. Neuron-specific expression of human angiotensinogen in brain causes increased salt appetite. Physiol. Genomics. 9:113-120. 